\title{
Development and Validation of a New Behavioral-Cognitive-Metacognitive Model for Explanation of SPD
}

\author{
Masoud Nikfarjam', Mehdi Rabiei ${ }^{2}$, Amir Mohsen Rahnejat ${ }^{3}$, Kazem Khorramdel ${ }^{4}$ \\ ${ }^{1}$ Medical Plants Research Center, Shahrekord University of Medical Sciences, Shahrekord, Iran \\ ${ }^{2}$ Department of Clinical Psychology, Baqiyatallah University of Medical Sciences, Tehran, Iran \\ ${ }^{3}$ Department of Clinical Psychology, AJA University of Medical Science, Tehran, Iran \\ ${ }^{4}$ Young Researcher and Elite Club, Electronic Branch, Islamic Azad University, Tehran, Iran \\ Email: ${ }^{*}$ rabiei_psychology@yahoo.com
}

Received 18 June 2015; accepted 2 August 2015; published 5 August 2015

Copyright (C) 2015 by authors and Scientific Research Publishing Inc.

This work is licensed under the Creative Commons Attribution International License (CC BY).

http://creativecommons.org/licenses/by/4.0/

(c) (i) Open Access

\begin{abstract}
The purpose of the present study is to provide an overview of the disorder and develop a new behavioral-cognitive-metacognitive model to explain it. The present study is a descriptive-correlational research which uses the structural equation model. In this study, at first, a comprehensive and detailed explanation of skin picking disorder is presented. Then the conceptual model is presented and finally, the model which is developed by using structural equation model is tested by Amos Graphics. The results of the structural equation model showed that the developed model in this study is able to explain skin picking disorder. Also the results of the overall fit indices for structural equation modeling show that the developed model has good fit to the sample data and is closely associated with the theoretical assumptions. Since the model presented in this study is multi-dimensional and takes into consideration all of the three behavioral, cognitive, metacognitive dimensions and these three dimensions are considered interdependent and not dependent and contradictory, it is considered as a new explanatory model. The model may prompt future researches into skin picking disorder and facilitate clinical treatments and case formulations associated with this disorder.
\end{abstract}

\section{Keywords}

Modeling, Skin Picking Disorder (SPD), Behavior, Cognition, Metacognition

\footnotetext{
${ }^{*}$ Corresponding author.
}

How to cite this paper: Nikfarjam, M., Rabiei, M., Rahnejat, A. M., \& Khorramdel, K. (2015). Development and Validation of a New Behavioral-Cognitive-Metacognitive Model for Explanation of SPD. Psychology, 6, 1223-1231. 


\section{Introduction}

Skin Picking Disorder (SPD) is a chronic problem which is characterized by excessive and recurrent manipulating and picking of the skin which is not due to dermatological conditions. Patients typically have desire and urge to pick their skin and it is difficult for them to control and stop these behaviors. Skin picking usually results in tissue damage and appearance deformity in these individuals (Snorrason, Belleau, \& Woods, 2012). Many of these patients suffer from functional impairments and emotional distress due to external deformity and inability to control these behaviors (Odlaug et al., 2013). Recently, SPD has been proposed as a separate diagnosis in the DSM-5 (The fifth edition of the Diagnostic and Statistical Manual for Mental Disorders) in the diagnostic category of Obsessive-Compulsive Disorder (OCD) and its associated disorders (Lochner, Grant, Odlaug, \& Stein, 2012; Rabiei, Donyavi, Nikfarjam, \& Mohammad Nezhady, 2015).

Literature review of mental disorders indicates that during the last twenty years, SPD has been under less clinical and special consideration than the other prevalent psychological disorders (Flessner \& Woods, 2006). In recent studies, the prevalence of SPD among general population of American adults has been reported between 1.4 to 5.4 percent (Hayes, Storch, \& Berlanga, 2009). The studies show that $75 \%$ of these disorders occur in females (Odlaug et al., 2013). Although so many people from general population consider skin picking as a part of their daily cleaning, but those who suffer from SPD carry out skin picking for a long period of time and their goal is to control and regulate emotions, feelings and unpleasant thoughts (Grant et al., 2012). Conducted studies on patients who were seeking treatment show that the range of damage to skin is from relatively slight to severe and it is often found in people who are looking for skin treatments such as plastic surgery, skin grafting, laser therapy, topical antibiotics or skin removing (Hlal, Barrimi, Kettani, Rammouz, \& Aalouane, 2014). SPD sufferers usually have problem with psychological and social performance such as anxiety and social avoidance, lack of effectiveness at school and in the workplace, depression and anxiety, and suicide ideation. SPD was not classified as a separate disorder in the official and authorized texts such as ICD-10 and DSM-IV-TR. The reason for this matter is not clear but likely it is because of the high comorbidity of SPD with a lot of other skin diseases (Lovato et al., 2012). But as mentioned earlier, this disorder classified as a separate disorder under the category of OCD disorders in DSM-5. SPD usually associated with disorders such as hair pulling (Trichotillomania), obsessive pulling and picking of body's hair, and body dysmorphic in which a person becomes obsessed with real or imaginary defects in their appearance or parts of the body (Lochner et al., 2012). Nail pulling or skin picking may be due to the dryness of skin or skin allergies or other skin disorders that cause itching. The picked parts should be carefully examined to ensure that picking is not due to sensitivity of the skin, skin infections or other skin diseases. Picking the skin of head and nose may also be due to head disorders. Certain types of liver diseases such as hepatitis can produce ulcers on the skin and these individuals may scratch these parts with nails and make the wounds apparent. After medical examinations, psychiatric evaluations should be performed. Skin picking behavior may be a behavioral response to boredom and impatience, fear and anxiety or stress that is caused by environmental stresses. The selected treatment for skin picking is behavioral management. Impatience, anxiety and avoidance behaviors must be managed through behavioral interventions (Stein et al., 2010). The clinical evidence of effective treatment methods for skin picking disorder is very rare. At present there is no coherent treatment package for skin picking but some satisfactory medical modals have been developed for it. For example, two clinical trials with placebo have shown that skin picking may have effective response to Fluoxetine. The Habit Reversal Training (HRT) has also been useful (Grant et al., 2012). Previous studies on SPD showed that the available evidence of etiology, incidence, duration and treatment for this disorder is very rare, and most studies included small groups or available non-clinical samples. Some studies suggested that treatments that are effective in obsessive-compulsive disorder can also be effective in this disorder. Cognitive Behavioral Therapy (CBT) can help to treat SPD. Acceptance and Commitment Therapy (ACT) may also be useful in treatment (Snorrason, Stein, \& Woods, 2013). Researches also indicate that SPD may be treated with SSRI drugs (Selective Serotonin Reuptake Inhibitors) such as fluoxetine, fluvoxamine and escitalopram effectively. Some studies represent that medical anti-seizure lamotrigine may be useful in the treatment of SPD (Stein et al., 2010). In addition to medical point of view, there are some other models for explaining and treatment of SPD that will be mentioned below. Based on the cognitive-behavioral perspective, SPD sufferers focus on their thoughts and feelings and misinterpret them and because of the wrong cognitive schemata, they consider them as a warning, and as a result they experience negative emotions and to reduce anxiety and discomfort due to negative thoughts, they engage in compulsive skin picking behavior (Denys, van Megen, \& Westenberg, 2003). The 
psychodynamic approach believes that aggressive and hostile desires towards others (through backlash and displacement) result in skin picking (Grant, Menard, \& Phillips, 2006). Some experts also believe that SPD is another form of the other mental disorders and mostly associated with depression and anxiety disorders and it is difficult to isolate these disorders from each other (Brakoulias, 2014). In the above-mentioned views, there is no clear examination and explanation regarding how SPD is started, continued and intensified. There is also no explanation regarding the relationship between the underlying psychological factors such as behavioral factors, cognitive factors and metacognitive factors. For example, in the cognitive view, it is believed that negative thoughts cause skin picking but there is no clear explanation for why negative thoughts don't result in SPD in all people.

In the present study, the behavioral-cognitive-metacognitive model is presented to explain skin picking. In this model, it is believed that cognitive factor can be starting point of the problem but to intensify the problem, it is in need of interacting with the metacognitive and behavioral factors. In other words, these factors are interdependent and they often don't act in isolation. To understand the model, we explain the proposed model in stages:In the behavioral-cognitive-metacognitive model, it is believed that at first negative cognitions come to the mind in which this phase is common in the mind and cognitive function of most people and it is a relatively normal phase, that is, the presence of negative cognitions (thoughts, impulses or images) don't mean the disorder. The results of some studies (e.g. Clark \& Beck, 2011; Freeston et al., 1997; Purdon \& Clark, 1993) also support this idea. This question may beraised that if negative cognitions don't cause the disorder by themselves, so which factors cause to the disorder? In the presented behavioral-cognitive-metacognitive model, the starting process, duration and exacerbation of SPD is explained as follows:

1) In this model, it is believed that our sight and attitude towards negative cognitions can result in cognitive distortion. For example, if we have negative attitude (negative metacognitive beliefs) towards negative cognitions of our mind, and consider them unnatural, after a while, we will have fear of them and consider them dangerous. Therefore, there is interaction between cognitive and metacognitive factors.

2) Considering these negative cognitions dangerous and scary will cause meta-worry. Worry may occur to many people and cause disorder, but meta-worry is more serious and usually leads people to the disorder. In this model, it is believed that negative beliefs towards negative cognitions result in meta-worry and then people decide to get rid of these negative cognitions in any way. In this phase, the individuals use thought control strategies or compulsive behaviors such as excessive skin checking and skin picking to reduce meta-worry (Rabiei, Salahian, Bahrami, \& Palahang, 2011; Donyavi, Rabiei, Nikfarjam, \& Mohmmadnezhady, 2015).

3) In order to not be faced with pain and discomfort due to negative thoughts, images, memories, impulses and emotions, individuals may do the following behaviors:

- Cognitive control strategies: all cognitive activities that aim to avoid the negative thoughts, images, memories and impulses are included in this category. In addition to the mechanisms that Freud and Anna Freud introduced, there are also some conscious strategies which are aimed at avoiding the negative thoughts, images, memories and impulses. Among these strategies are distracting, concentration on the thoughts, positive images, memories and impulses and positive assessment which may cause individual's temporary calmness, but in fact cause individuals to get away from accepting the realities of their existence.

- Behavioral control strategies: in order to avoid being faced with negative thoughts, images, memories, impulses, emotions and behaviors, we do various actions and behaviors, because they are painful. In addition to avoidance, safety and obsessive behaviors such as skin picking, all the strange behaviors of mental disorders are also a part of these behaviors.

A review of the related literature on the explanation of SPD shows that different models have been presented, but most of the models are disable to explain and predict SPD or they are not holistic and multidimensional. The presented model in this study has been developed to compensate a part of these deficiencies. The main objective of the present study is to develop and validate a behavioral-cognitive-metacognitive model to explain SPD. Among the secondary objectives of the study were the determination of the relationship between cognitive factor and metacognitive and behavioral factors, determination of the role of metacognitive factor as a mediator between cognitive and behavioral factors, and finally determination of the goodness of fit for the developed model.

\section{Method}

The present study is a survey research for designing a model. The model presented in this study has been developed based on the authors' researches and clinical studies (Rabiei, Mulkens, Kalantari, Molavi, \& Bahrami, 
2012; Rabiei, Nikfarjam, Movassagh, \& Goldoz-Khayat, 2014; Donyavi, Rabiei, Nikfarjam, \& Rahnejat, 2015) and it has theoretical and clinical background.

All mentioned variables in the model of the present study have face and content validity and they are wellmatched to the behavioral-cognitive-metacognitive models. That is, there is no difference between the definition of the variables in the present study and the definition presented by cognitive, behavioral and metacognitive experts. Structural equation model was used to assess validity of the structure and fitness of model to data.

The statistical population of the study included all individuals who referred to counseling centers and medical clinics in Isfahan in 2013. The sample of the study was selected from counseling centers and medical clinics by cluster sampling. Since there are also SPD sufferers in the non-clinical sample and also in order to assess fitness of the model in larger sample and due to correlational analysis and structural equation model, the sample was selected from non-clinical and outpatient population. To determine size of the sample, sample size estimation method of Cochran was used. Due to the fact that in this study, the actual number of statistical population (N) was unknown, and as a result the standard deviation of population (S) was also unknown and undetectable, therefore the formula that is based on the square root of sample's standard deviation has been used to estimate the sample size. In this regard, given the uncertainty of the standard deviation of population (S), it is required to select preferably a sample of at least 30 people to estimate the standard deviation of sample (Kerlinger, 2000). Thus to determine the sample size, at first a primary sample of 40 people was selected and the obtained variance with an accuracy coefficient of 0.05 was placed in the Cochran formula. The results showed that a sample of 600 people is sufficient and appropriate for the present sample. However, 640 people were selected as a sample to don't be any problem in the case of loss of questionnaires and difficulties in completing the questionnaires. Five questionnaires were excluded from the sample because they were not complete and finally a sample of 635 people was analyzed. Having at least an education level of diploma, no hospitalization, being from Isfahan, being Persian, being at the age range of 15 - 50 and voluntary cooperation of the participants were among the criteria of the present study. The people who didn't have the above-mentioned criteria were excluded from the study.

To verify the developed model, AMOS was used. Pearson correlation and multiple-regression were used to analyze the correlational hypotheses, and overall fit indices, and Parsimony and comparative indices were used to evaluate the fitness of the proposed model. The reason for selecting the structural equation model to assess validity of the conceptual model is that this model is superior to exploratory analysis in terms of reducing the amount of error, increasing the purity of the factors and the power of fitness evaluation of the overall model. Furthermore, the confirmatory factor analysis also includes the exploratory analysis.

In this study to observe moral considerations, it is explained to the participants that they are going to take part in a research which aims to construct and evaluate a new treatment model on obsession symptoms. The clients were also assured that their information will be completely confidential. Moreover, along with answering to all questions of the participants about the research, they were assured that the results of the study will be published statistically as an overall conclusion and not individually and they are free to take part in the study or not and in any stage of the study they can quit the study.

\subsection{Instruments}

\subsubsection{Skin Picking Scale (SPS)}

Skin Picking Scale (SPS); (Keuthen et al., 2001) is a 6-item self-rating scale which ask about skin picking symptoms such as (desire and urge to skin picking, the time taken for skin picking, control on skin picking pressures, skin picking behaviors' interventions on one's social and occupational performance, distress associated with skin picking and avoidance behaviors due to skin picking). The questions are on a Likert-scale from 0 to 4 score and the total score range is from 0 to 24 . Good internal consistency $(\alpha=0.8)$ and acceptable testretest reliability $(r=0.9)$ has been reported (Sulkowski et al., 2011). SPS is used in clinical and non-clinical samples. The convergent validity of the scale supports SPS and indicates high correlation of the scale with other skin picking scales in non-clinical samples (Sulkowski et al., 2011). In Iran the Persian version of SPS has been normalized by (Rabiei, 2014). Their results have showed that Persian version of SPS has good reliability and validity.

\subsubsection{Cognitive Distortions Scale (CDS) (Briere, 2000)}

This questionnaire measures the cognitive distortions which include: a) self-blame, b) helplessness, c) disappointment, d) mental preoccupation with danger and bad events. The questionnaire includes 40 items. The par- 
ticipants responded on a five-point scale (from completely true to completely wrong). The score range of each respondent varies from 0 to 200. The Cronbach's alpha of the questionnaire is reported above 0.97 (Briere, 2000). In the present study the reliability and validity of this questionnaire was calculated. The results showed that CDS has appropriate validity (convergent and divergent) and reliability (Cronbach’s Alpha) in Iranian sample.

\subsubsection{Dysfunctional Attitudes Scale (DAS)}

This questionnaire is a self-report scale which has been developed by (Weissman, 1978) to evaluate attitudes, schemata and negative beliefs. This scale is one of the most well-known instruments in the field of cognitive researches which has 40 statements graded on a 7 point Likert-scale. The scale measures core and intermediate beliefs. Reliability coefficient of the scale using Cronbach's Alpha and test-retest coefficient have been reported 0.9 and 0.73 respectively. This scale includes four underlying factors of perfectionism, the need for approval of others, the need for satisfying others and evaluation of performance.

\subsubsection{Automatic Thoughts Questionnaire (ATQ) (Hollon, 1980)}

The ATQ is a 30-item questionnaire developed to measure the frequency of occurrence of automatic negative thoughts. In this questionnaire, there are 30 negative self-statements which are linked to depression, and individuals must express experiences of their thoughts over the last week. Scoring is done on a Likert-scale from never (1) to always (5). Internal consistency and test-retest reliability has been obtained more than 0.8 (Ghassemzadeh, Mojtabai, Karamghadiri, \& Ebrahimkhani, 2005).

\subsubsection{Meta Cognitions Questionnaire (MCQ)}

The short form of MCQ (Cartwright-Hatton, 2004) has been revised to measure metacognative beliefs. This questionnaire has 30 items and each respondent chooses one of the options of (disagree, somewhat agree, agree, strongly agree). These options will be scored 4, 1, 2, and 3 respectively. The 30-item Metacognitions questionnaire measures 5 dimensions of metacognitive beliefs and processes: 1) cognitive confidence 2) positive beliefs about worry 3) cognitive self-consciousness 4) negative beliefs about uncontrollability and danger 5) beliefs about need to control thoughts. Cronbach's alpha Coefficient of the questionnaire and its components has been reported on a range of $0.72-0.93$. The test-retest reliability coefficient of the questionnaire is 0.73 .

\subsubsection{Obsessive-Compulsive Behavior Scale (OCS)}

This scale is a self-test with 8 items which shows problematic behaviors in obsessive compulsive disorders. The test which is constructed by the researcher took a form of 5-point Likert scale and scored from 0 to 4 and includes safety and neutralizing behaviors, avoidance behaviors and obsessive behaviors. Psychometric characteristics of the questionnaire were obtained in the study in which the Cronbach's alpha and test-retest reliability (with 3 weeks interval) was 0.87 and 0.81 respectively. The test also has good face and content validity, and investigating construct validity by using exploratory factor analysis with final Varimax rotation showed 4 factors of safety and neutralizing behaviors, avoidance behaviors and obsessive behaviors which in sum explained 68\% of the questionnaire's variance.

\section{Results}

To analyze the model and to evaluate its validity, the structural equation model was used by Amos Graphic. With regard to the objectives of the study, the model and its conceptual explanation has been presented in this part. In order to assess validity of the model, the standard coefficients of the model and model's fitness index is also presented:

As represented in the Figure 1, core beliefs affect cognitive distortions through intermediate beliefs (rules, assumptions and attitudes). Negative metacognitive beliefs affect negative automatic thoughts through cognitive distortions and increase their influence, and intermediate beliefs affect negative automatic thoughts through cognitive distortions and increase them. Cognitive distortions also affect metaworry through the mediation of negative automatic thoughts and increase its influence. Furthermore, negative automatic thoughts affect thought control strategies through the mediation of metaworry and increase them and affect and increase obsessive behaviors through metaworry and finally metaworry and thought control strategies affect obsessive disorder through the mediation of obsessive behaviors. In general, the results show that there is an interaction between 


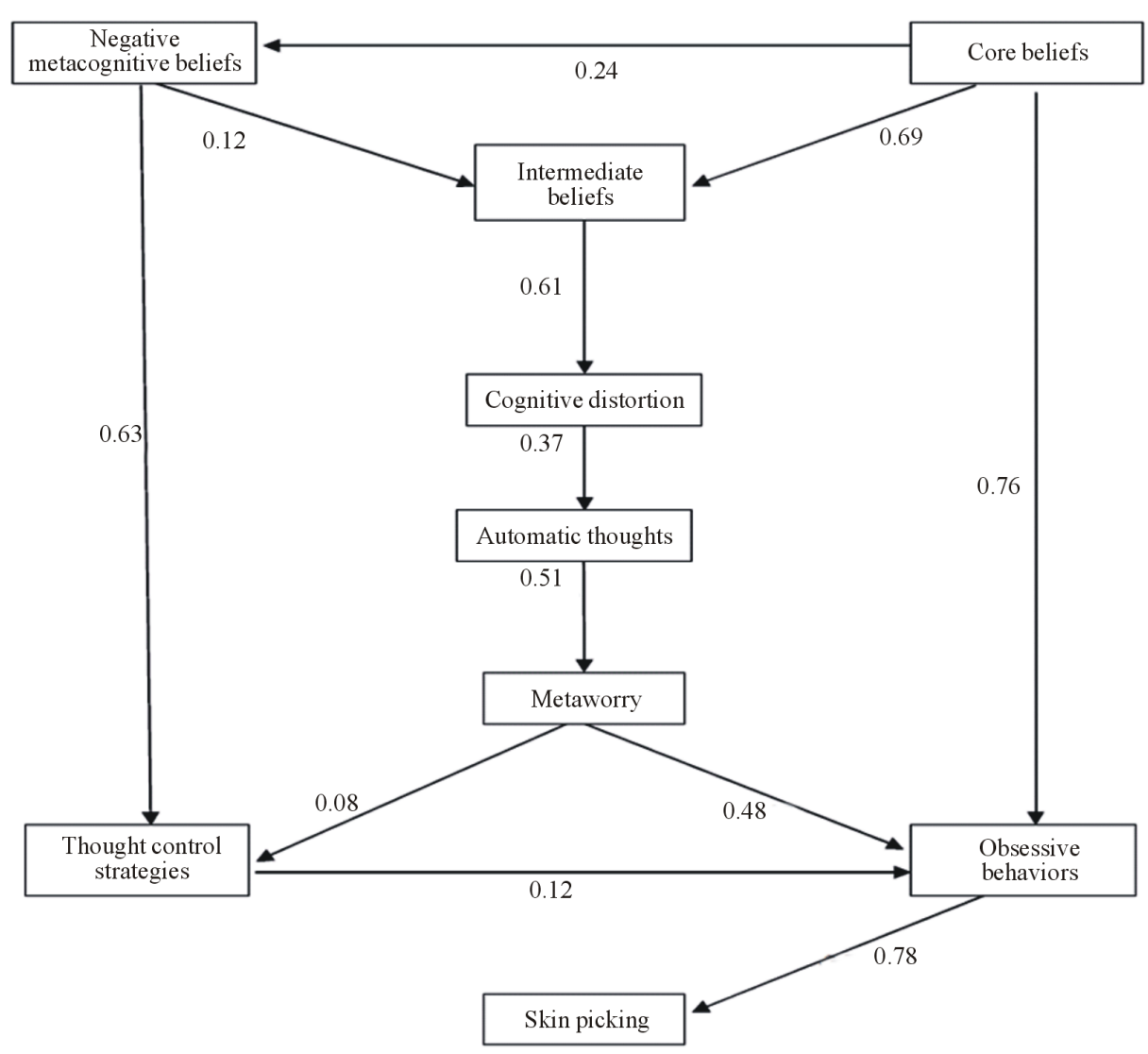

Figure 1. Conceptual model of the research and its standard coefficients.

the cognitive and metacognitive factors and metacognitive factor plays a mediating role between the cognitive and behavioral factors. Therefore the hypotheses of the study are consistent with the objectives of the study and they are approved.

In the above model chi-square value has not been significant after correction $\left(P=0.001, X^{2}=1.23\right)$ (Table 1 ). This result indicates that the model is acceptable. Root Mean Square Error of Approximation (RMSEA) which is one of the model's fitting indices is 0.01 and Goodness of Fit Index (GFI) is also above 0.9 which shows that the model has appropriate fitting. Comparative Fit Indices (CFI) and Tucker-Lewis fit Index (TLI) values are also above 0.9 which confirms the model. Parsimony indices of the model are not provided in the table, but PCFI and PNFI values are 0.83 and 0.88 respectively in which these values add model's confirmation. $P$ close value is reached to 0.18 which the values above 0.10 are good for the model.

\section{Discussion}

This study aimed to develop and validate a behavioral-cognitive-metacognitive model to explain SPD. To this end, based on the theoretical and experimental history and clinical experiences of the authors, the target model was developed and tested for its validity. In this model, the behavioral components (avoidance, obsessive and safety behaviors) - the cognitive components (core beliefs, intermediate beliefs, automatic thoughts) - the metacognitive components (positive and negative metacognitive beliefs and metacognitive strategies) have been considered in explaining SPD. The findings of the study are consistent with (Stein et al., 2010) in terms of the relationship between cognitive components and SPD. Among the reasons for this relationship, it can be noted that in these patients the underlying beliefs are more general, inflexible, rigid, negative and irrational that cause mind's natural processes associate with cognitive distortions, leading to an increase in negative automatic thoughts in these patients. This finding is consistent with the results of (Wells, 2009) and (Ghahvehchi-Hosseini, 2013) in the case that positive beliefs cause an increase in negative thoughts. However (Wells, 2009) didn't mention that metacognitive components have an interaction with cognitive and behavioral factors with regard to 
Table 1. Fitness indices of the hypothetical model conformingto the figure.

\begin{tabular}{cccccc}
\hline RMSEA & RMR & TLI & GFI & CFI & AGFI \\
\hline 0.013 & 0.02 & 0.89 & 0.92 & 0.91 & 0.93 \\
\hline
\end{tabular}

affecting the emotional disorders such as obsession, and his results are consistent with the results of (Keuthen et al., 2001; Stein et al., 2010; Zuchner, 2006) in terms of the relationship between behavioral components and SPD. Among the reasons for this relationship may be noted that the obsessive, avoidance and safety behaviors and the neutralizing behaviors in the patients cause underlying and more general, inflexible, rigid, negative and irrational beliefs and problematic metacognitive beliefs don't be corrected and cause natural processes of mind associate with the cognitive distortions, and the negative automatic thoughts be continued and intensified.

In general, it can be concluded that because CBT approach takes into consideration only the cognitive and behavioral aspects and ignores metacognitive aspects such as metaworry and thought control strategies, has some deficiencies and limitations. On the other hand, metacognitive approach also has some weaknesses and limitations because it takes into consideration only the key aspects and has less emphasis on behavioral and cognitive aspects (such as core beliefs, intermediate beliefs, cognitive distortions and negative automatic thoughts). The developed model in the present study takes into consideration all the three behavioral, cognitive and metacognitive aspects and in this model it is believed that these three aspects are interdependent and have interaction with each other in the starting point and duration of SPD. As depicted in the model, the cognitive factors such as core beliefs and negative automatic thoughts cause an increase in worry and then followed by metaworry and these factors cause an increase in the obsessive behaviors and thought control strategies. Whereas the cognitive view believes that the cognitive factors may cause the disorder by itself.

In the model developed in this study, it is believed that negative cognitions can be problematic when they cause metaworry. Metaworry occurs when one fears from his/her negative cognitions and considers them abnormal and special to his/her own mind. Wells (2009) believes that when people are afraid of their thoughts and consider them abnormal and uncontrollable, they frighten themselves more and at the same time try to eliminate and control their thoughts due to worry and fear. However efforts to control and eliminate the negative thoughts don't cause a decrease in the negative thoughts and impulses, but cause failure in controlling thoughts, and sometimes increase the negative thoughts. When one disappoints to control the negative thoughts, s/he resorts to the obsessive, avoidance and safety behaviors to reduce his/her anxiety and stress. The obsessive and avoidance behaviors reduce anxiety temporarily and this issue encourages individuals to use the obsessive behaviors frequently and compulsorily, but this action lead to the retention of obsessive behaviors in the long run and result in duration of the disorder. According to the model of this study, skin picking behavior is enjoyable because it causes distraction from the pain of negative thoughts and is considered as a strategy to control negative thoughts and it cause to duration of the negative thoughts and skin picking obsession. Therefore, in explanation of SPD from the developed model's perspective, all the three behavioral, cognitive and metacognitive aspects and their interactions have been taken into consideration.

Among the limitations of this study are as follows:

In the developed model, only behavioral, cognitive and metacognitive dimensions were targeted and the other dimensions such as environmental, social-local, familial, economical, and spiritual and other factors have been neglected and have not been the target of the study. To develop the model, only general and non-clinical participants were employed, that is, the method has been correlational and there was no possibility for an Ex-Post Facto research.

The present study has been carried out on non-clinical population and it is necessary to test the developed model quantitatively and qualitatively in longitudinal and experimental studies on patients who suffer from skin picking obsession and if possible, be compared with the existing models.

\section{Acknowledgements}

The authors would also like to acknowledge the assistance of participants and all of the clinicians and research assistants who conducted the assessments. 


\section{Declaration of Conflicting Interest}

The authors declare that there are no conflicts of interest.

\section{Funding}

This research received no specific grant from any funding agency in the public, commercial, or notfor-profit sectors.

\section{References}

Brakoulias, V. (2014). DSM-5 Bids Farewell to Hypochondriasis and Welcomes Somatic Symptom Disorder and Illness Anxiety Disorder. Australian \& New Zealand Journal of Psychiatry, 48, 688. http://dx.doi.org/10.1177/0004867414525844

Briere, J. (2000). The Cognitive Distortion Scale Professional Manual. Psychological Assessment Resources.

Cartwright-Hatton, S., M. A., Illingworth, V., Brocki, J., Harrington, R., \& Wells, A. (2004). Development and Preliminary Validation of the Meta-Cognitions Questionnaire-Adolescent Version. Journal of Anxiety Disorders, 18, 411-422. http://dx.doi.org/10.1016/S0887-6185(02)00294-3

Clark, D. A., \& Beck, A. T. (2011). Cognitive Therapy of Anxiety Disorders: Science and Practice. New York: Guilford Press.

Denys, D., van Megen, H. J., \& Westenberg, H. G. (2003). Emerging Skin-Picking Behaviour after Serotonin Reuptake Inhibitor-Treatment in Patients with Obsessive-Compulsive Disorder: Possible Mechanisms and Implications for Clinical Care. Journal of Psychopharmacology, 17, 127-129. http://dx.doi.org/10.1177/0269881103017001718

Donyavi, V., Rabiei, M., Nikfarjam, M., \& Mohmmadnezhady, B. (2015). Body Image and Meta-Worry as Mediators of Body Dysmorphic Disorder. International Journal of Clinical Medicine, 6, 170-176. http://dx.doi.org/10.4236/ijcm.2015.63022

Donyavi, V., Rabiei, M., Nikfarjam, M., \& Rahnejat, A. (2015). Develop and Validate a Metacognitive-Cognitive-Behavioral Model for Body Dysmorphic Disorder. Open Journal of Medical Psychology, 4, 45-52. http://dx.doi.org/10.4236/ojmp.2015.42005

Flessner, C. A., \& Woods, D. W. (2006). Phenomenological Characteristics, Social Problems, and the Economic Impact Associated with Chronic Skin Picking. Behavior Modification, 30, 944-963. http://dx.doi.org/10.1177/0145445506294083

Freeston, M. H., Ladouceur, R., Gagnon, F., Thibodeau, N., Rheaume, J., Letarte, H., \& Bujold, A. (1997). Cognitive-Behavioral Treatment of Obsessive Thoughts: A Controlled Study. Journal of Consulting and Clinical Psychology, 65, 405-413. http://dx.doi.org/10.1037/0022-006X.65.3.405

Ghahvehchi-Hosseini, F. (2013). Meta-Cognitive Therapy versus Cognitive Therapy in Reducing Meta-Worry in Students with Test Anxiety. Journal of Behavioural Science, 4, 1-8.

Ghassemzadeh, H., Mojtabai, R., Karamghadiri, N., \& Ebrahimkhani, N. (2005). Psychometric Properties of a Persian-Language Version of the Beck Depression Inventory—Second Edition: BDI-II-PERSIAN. Depress Anxiety, 21, $185-192$. http://dx.doi.org/10.1002/da.20070

Grant, J. E., Menard, W., \& Phillips, K. A. (2006). Pathological Skin Picking in Individuals with Body Dysmorphic Disorder. General Hospital Psychiatry, 28, 487-493. http://dx.doi.org/10.1016/j.genhosppsych.2006.08.009

Grant, J. E., Odlaug, B. L., Chamberlain, S. R., Keuthen, N. J., Lochner, C., \& Stein, D. J. (2012) Skin Sicking Disorder. The American Journal of Psychiatry, 169, 1143-1149. http://dx.doi.org/10.1176/appi.ajp.2012.12040508

Hayes, S. L., Storch, E. A., \& Berlanga, L. (2009). Skin Picking Behaviors: An Examination of the Prevalence and Severity in a Community Sample. Journal of Anxiety Disorders, 23, 314-319. http://dx.doi.org/10.1016/j.janxdis.2009.01.008

Hlal, H., Barrimi, M., Kettani, N., Rammouz, I., \& Aalouane, R. (2014). Factitious Disorder and Skin Picking: Clinical Approach: A Case Report. Encephale, 40, 197-201.

Hollon, S. D., \& Kendall, P. C. (1980). Cognitive Self-Statements in Depression: Development of an Automatic Thoughts Questionnaire. Cognitive Therapy and Research, 4, 383-395. http://dx.doi.org/10.1007/BF01178214

Kerlinger, F. N. (2000). Foundations of Behavioral Research. San Diego, CA: Harcourt College Publishers.

Keuthen, N. J., Wilhelm, S., Deckersbach, T., Engelhard, I. M., Forker, A. E., Baer, L., \& Jenike, M. A. (2001). The Skin Picking Scale: Scale Construction and Psychometric Analyses. Journal of Psychosomatic Research, 50, 337-341. http://dx.doi.org/10.1016/S0022-3999(01)00215-X

Lochner, C., Grant, J. E., Odlaug, B. L., \& Stein, D. J. (2012). DSM-5 Field Survey: Skin Picking Disorder. Annals of Clinical Psychiatry, 24, 300-304. 
Lovato, L., Ferrao, Y. A., Stein, D. J., Shavitt, R. G., Fontenelle, L. F., Vivan, A., \& Cordioli, A. V. (2012). Skin Picking and Trichotillomania in Adults with Obsessive-Compulsive Disorder. Comprehensive Psychiatry, 53, 562-568. http://dx.doi.org/10.1016/j.comppsych.2011.06.008

Odlaug, B. L., Lust, K., Schreiber, L. R., Christenson, G., Derbyshire, K., \& Grant, J. E. (2013). Skin Picking Disorder in University Students: Health Correlates and Gender Differences. General Hospital Psychiatry, 35, 168-173. http://dx.doi.org/10.1016/j.genhosppsych.2012.08.006

Purdon, C., \& Clark, D. A. (1993). Obsessive Intrusive Thoughts in Nonclinical Subjects. Part I. Content and Relation with Depressive, Anxious and Obsessional Symptoms. Behaviour Research and Therapy, 31, 713-720.

Rabiei, M. (2014). Factor Structure, Validity and Reliability of the Revised Version of Skin Picking Scale. Zahedan Journal of Research in Medical Sciences, 9, 40-44.

Rabiei, M., Donyavi, V., Nikfarjam, M., \& Mohammad Nezhady, M. A. (2015). Validation of a Classification System for Obsessive-Compulsive and Related Disorders Based on DSM-5. Open Journal of Psychiatry, 5, 137-143. http://dx.doi.org/10.4236/ojpsych.2015.52015

Rabiei, M., Mulkens, S., Kalantari, M., Molavi, H., \& Bahrami, F. (2012). Metacognitive Therapy for Body Dysmorphic Disorder Patients in Iran: Acceptability and Proof of Concept. Journal of Behavior Therapy and Experimental Psychiatry, 43, 724-729. http://dx.doi.org/10.1016/j.jbtep.2011.09.013

Rabiei, M., Nikfarjam, M., Movassagh, M. T., \& Goldoz-Khayat, N. (2014). The Effectiveness of Metacognitive-CognitiveBehavioral Intervention on Severity of the Symptoms of Hair Pulling Disorder. Journal of Behavioral Sciences, 7, 373378.

Rabiei, M., Salahian, A., Bahrami, F., \& Palahang, H. (2011). Construction and Standardization of the Body Dysmorphic Metacognition Questionnaire. Journal of Mazandaran University of Medical Sciences, 21, 43-52.

Snorrason, I., Belleau, E. L., \& Woods, D. W. (2012). How Related Are Hair Pulling Disorder (Trichotillomania) and Skin Picking Disorder? A Review of Evidence for Comorbidity, Similarities and Shared Etiology. Clinical Psychology Review, 32, 618-629. http://dx.doi.org/10.1016/j.cpr.2012.05.008

Snorrason, I., Stein, D. J., \& Woods, D. W. (2013). Classification of Excoriation (Skin Picking) Disorder: Current Status and Future Directions. Acta Psychiatrica Scandinavica, 128, 406-407. http://dx.doi.org/10.1111/acps.12153

Stein, D. J., Grant, J. E., Franklin, M. E., Keuthen, N., Lochner, C., Singer, H. S., \& Woods, D. W. (2010). Trichotillomania (Hair Pulling Disorder), Skin Picking Disorder, and Stereotypic Movement Disorder: Toward DSM-V. Depress Anxiety, 27, 611-626. http://dx.doi.org/10.1002/da.20700

Sulkowski, M. L., Mancil, T. L., Jordan, C., Reid, A., Chakoff, E., \& Storch, E. A. (2011). Validation of a Classification System of Obsessive-Compulsive Spectrum Disorder Symptoms in a Non-Clinical Sample. Psychiatry Research, 188, 65-70. http://dx.doi.org/10.1016/j.psychres.2011.01.015

Weissman, A. N., \& Beck, A. T. (1978) Development and Validation of the Dysfunctional Attitude Scale: A Preliminary Investigation. Proceedings of the 62nd Annual Meeting of the American Educational Research Association, Toronto, 27-31 March 1978.

Wells, A. (2009). Metacognitive Therapy for Anxiety and Depression. New York: Guilford Press.

Zuchner, S., Cuccaro, M. L., Tran-Viet, K. N., Cope, H., Krishnan, R. R., Pericak-Vance, M. A., Wright, H. H., \& AshleyKoch, A. (2006). SLITRK1 Mutations in Trichotillomania. Molecular Psychiatry, 11, 887-889.

http://dx.doi.org/10.1038/sj.mp.4001898 